\title{
Bengt Engan:
}

\section{Exploring reality through new lenses}

The informal essay as a an academic genre

Paper for the conference Civil society, social capital and social work December 13th-17th, 2004.

University of Havana, Cuba 
an the essay genre used in education and reserarch provide new options for describing reality? This genre has already obtained a strong position in philosophy, in which it

has proven to be appraopriate when dealing with "the far too big and the far too small questions" ${ }^{1}$. But what about the essay as a supplement to - or even a replacement for traditonal descriptions within social science in higher education?

In Norway the essay genre is now used in quite a number of higher studies during recent years. This is especially the case in various professional studies oriented towards a practice field. In this paper I will try to illuminate (1) the limitations there might be to traditional scientific genres like the article and the thesis; (2) how the essay may provide possibilities for new textual and meaning structures within alternative hermeneutic paradigms, and finally (3) how an enhanced reportoire of genres may be connected to an enhanced reportoire of ways of learning and comprehension.

1.

Within scientific writing one tries to minimize the significance of "form", compared to "content". The linguistic ideal in a scientific article or thesis is the unmarked normal prose, in which the writing subject to take care of objectivity and validity is supposed to stay in the background. Thus the language should be transparent, so to speak, and should not "get in the way" of content. But linguistic practice will always reflect who is saying what to whom about what, in what context, and with what intention. This is an ancient insight forming the foundation of rhetoric as a discipline; talking and writing always means using rhetoric techniques.

It is quite understandable and legitimate that scientists focus on keeping distance from the subjective, expressive, literary language, in order to emphasize the crucial difference between facts and fiction. At the same time, the social sciences aim to describe the complexity and dynamics of the phenomena we investigate. When scientific projects insist that the object of research should be grasped in a strict categorical system of concepts, we may see that this (which is also a certain "form") reduces and masks important aspects of reality. This is especially true when the researcher adopts alternative approaches to that of the logicalpositivist paradigm and uses strategies such as ethnography, life-stories and participant observation. In this kind of social interaction, relevant research data may appear to be the researcher's own personal experience as an (inter)acting subject, rather than data built upon observation, interviews and quantitative mapping.

The issue is then how can personal experience be given a linguistic processing and genre that enables us to present them as valuable scientific products? Language appears with a double nature: on the one hand it gives us the opportunity to categorize phenomena, processes and relations in the world that surrounding us. This enables us to comprehend phenomena cognitively, and to make them subject to experience as well as analysis and abstraction. But language is also a medium, located between us and reality, which means that language defines what falls within the realm of our perception and our comprehension. Thus our understanding of reality cannot get more "real" than the language itself allows!

This is of course an important topic within philosophy, in which one has been greatly interested in the relationship between language and acknowledgement. But reflecting on the use of language and identifying problems related to language is also essential within any epistemological framework and science theory in general. Hence it is something that researchers and educators - for instance within the social sciences - must relate to, if their aim is to get a meta perspective on their own and other diciplinary practices. The debate on

${ }^{1}$ Quoting the Norwegian essayist Georg Johannessen 
the use of language in relation to the production and mediation of scientific knowledge is usually not focused on language per se. What is questioned is rather the level of accuracy of words and concepts, readability, and the like. This is of course important in itself, but I would like to extend the perspective towards the major structures at work in the text, namely the genre within which the text works.

In textual science the concept of genre holds a central position, because it refers to quite basic conditions as to how a text can work in a communicative setting. While the popular perception of the concept genre is something like "category of texts", textual science claims that a genre is basically a social category regulating the relationship between writer, reader and the object of the text (the "referent") ((Berge 2003). This is due to perspectives over the last decades concerning the social function of language, as formulated in the so called systemic-functional grammar, where language (similar to, and interacting with other sign systems) performs semiotic "work", for which the aim and major meaning is to be found at a higher, social level (Halliday 1985). In the same way that the researcher within hermeneutic methodology must reflect upon himself as an interpreting subject in a certain context, textual science states that all communication is intersubjective, it is something that takes place between interpreting subjects. The textual meaning is not transmitted, it is something that is created twice, so to speak - as the writers text, with a potential of meaning, and as the reader's text, with a realized meaning (Linell 1995, Wells 1999).

The essay stems directly from the renaissance. It is the only genre that can be traced back to one specific person and one specific moment in history: In 1580 the Frenchman Michel de Montaigne (1533-1592) was the author of a book called Les Essais, and thereby a new genre was actually created! With his subjective, literate style, combined with profound reflections, he apparently touched something inportant in his contemporary surroundings - Les Essais soon became a model for many writers. Montaigne claimed that personal experience should be the base and point of departure for all true acknowledgement. Leaning to others' thoughts and experience, as this is expressed by the written word, was not sufficient. As a thinker and philosopher, the writer would rather move into real life, collect impressions and then use these as the raw material for reflections on both the very largest questions and the (apparently) more trivial aspects of what it means to be a human. "Essais" means something like "attempt", and connotes that the writer would like to appear humble to his/her topic. Rather than draw firm conclusions he/she should illuminate the state of a problem, and thus hopefully guide the reader a bit further in his/her comprehension of the world. We should also note that the essay traditionally had a rather exclusive appearance, because the writer assumes that there is a reader at approximately the same educational level as himself. Thus an essayistic text does not necessarily address the general public. These are some properties in the tradition of what we call the French, "informal" essay.

While Montaigne should be honored for being the pioneer, the English philosopher Francis Bacon (1561-1626) soon followed up. He developed what eventually turned into the AngloAmerican variety: the "formal" essay, with its somewhat stronger demands to structure and clear argument. In this tradition, the essay label is used on quite a number of sub genres containing scientific, reasoned texts. However, in Norway, as in Central Europe, the "French" essay has always had the strongest appeal, or to put it another way: to call yourself an essay writer, you should stick to the Montaigne tradition! Thus the combination of personal experience, expressed in the shape of a narrative, and the ability to reflect upon this experience generates today's brand of the informal essay. The basic idea is that describing complex relations between humans, between individual and community, between culture and nature, between present, past and future requires another way of expressing yourself than the strict logicaldiscourse argument can offer. Relationships deal with events and processes, i.e. something and someone taking place in a complex, more or less vague totality in a field of practice. It is often not possible to reduce such complexities to scientific categories, they may be more 
accurately described though the use of the narrative. So the epic form, with its chronology and historical analysis, may prove to be the best way of taking care of the qualities in this kind of description of events.

However, the narrative could become just an shallow registration of events, which we may find entertaining and worth reading, but not possessing significant scientific value. What separates the essay from the pure epic genres is that the narrative should serve as the starting point for the writer's reflections. The story thus becomes part of a bigger and somewhat more ambitious project: to create a totality of events, ideas and inferences that neither the narrative nor the reasoning separately is capable of expressing. In this sense, there is a reason why some of the most outstanding of our contemporary essay writers use their impressions from journeys as the base for their reflections. The impressions on a journey are often overwhelming, but using them as the raw material for writing does not necessarily make texts suitable meeting scientific or philosophical standards. To gain an impression of the difference between ordinary, anecdotal travel stories and essayistic approaches, it should suffice to read some pages from the production of the Polish journalist Ryszard Kapu_i_ski and the Danish writer Carsten Jensen...

\section{2.}

In a story the axis of time is the most important structuring principle: the events take place in a certain sequence, and this is what gives each of them meaning. In a typical scientific article we will, likewise, find a dominating sequential logical structure mainly consisting of premisesconclusions and cause-effect relationships. These seem to be very different genres, but they are still both based upon strict linearity, meaning that different content elements must appear in a certain order to work within the text as a whole.

Contrary to this, we tend to think in a way that is more based on spontaneous shifts, associations and even digressions. This is often called brain storming and mindmaps. What we regard to be creativity is often nothing but finding such new and unexpected relations between elements previously known. Besides, reality as we perceive it is not necessarily coherent. Phenomena often appear to be contradictory and paradoxical, and are hard to interpret, let alone to explain. The essay genre does not insist that there is always rational and logical relationships in our experience, but rather allows for a more fragmentary description. The essay invites, in other words, the writer to combine a linear and more associative textual structure. One major theme will carry the text as a whole, but it is possible to combine this with a rich hierarchy of subthemes and "excursions" within a topic. An informal essay thus tends to appear more as an open and dialogical text, an invitation to the reader to participate in a sequence of notions and inferences on a subject. At the same time the text is basically a unique, highly personal and independent product, that quite obviously could only have been written by this person.

One interesting analogue to this would be the way information is organized on the internet (in particular the World Wide Web), where the leaps from "place" to "place" in a fragmented "docuverse" 2 is more typical than long, coherent texts. The so called hypertext provides a dimension in which potential coherence and meaning is found at a higher level (a "hyper"level) and not within each appearance ("node") of texts. To navigate in this information universe has therefore become a crucial part of todays literacy.

The ability to build a comprehensive, coherent reasoning will of course still be crucially important within the field of academic writing. But depending on the topic, the statement of problems, the complexity of the issues being dealt with, and the competence of the writer, an alternative genre such as the informal essay may prove to offer new epistemological approaches, as well as new esthetic qualities.

2 This concept stems from Theodore H. Nelson, who also invented the term "hypertext" in 1965. 
The focus on the essay as an academic genre in Norway - especially in student writing - is related to the expansion and change of both objects of research and the disciplinary portfolio in higher education. During the last decade a number of professional studies have been gradually more "academized": the nursing profession, the social work profession and journalism, just to mention a few. Being part of a major, nation wide system of academic standards, these studies have had to adapt to disciplinary and pedagogical traditions that seem somewhat unfamiliar. Because of this, researchers and educators in this realm have been engaged in identifying and emphasizing the uniqueness of professional studies. This means focusing on professional practice, both as a learning context and as a research object. We are here again dealing with phenomena, processes and situations that contain many physical, technological and social relationships, i.e. so many variables that it is difficult to give an unambiguous, causal description of what is going on. The essay has frequently proved to be an appropriate way of expression when this kind of "practice stories" are to be mediated and made the object for analysis and reflection.

The essay thus expands the repertoire of ways to use language and process the impressions that occur when encountering practice. This consciousness concerning different reality perspectives and how we can apply appropriate ways of work and expressions to take care of them also corresponds to the notion of learning styles in pedagogy. The American researchers Rita and Ken Dunn are considered to be originators of a theory on what individual differences between students implies, in terms of how they actually learn (Dunn and Dunn 1972). One important issue they raise is the dichotomy between analytic and holistically oriented persons. Some people are capable of (and prefer) working with limited categories, analytical models, unambiguous descriptions of systems, and a clear, linear progression. Others prefer to focus on totality and major aims from the first moment in a learning session, and they are capable of working with synthesis and information presented in a random, non-linear order. However, while the educational system is usually oriented towards - and encourages - the analytical way of approach, researchers belonging to the learning style theory claim that the population consists of twice as many "holists" as "analysts" (approx. 50\% vs. 25\%) (Dunn et al. 1995). The last quarter of the population is able to apply both kinds of approach, they thus have what is called a "flexible learning style".

In this perspective it becomes even more interesting to consider what the essay genre can offer - for the single student and researcher, as well as for the disciplinary community as a whole. If we start considering students' personal experience as a resource in a scientific context, this may lead to exciting methodological and epistemological implications. The essay then becomes more than just one among many ways of presenting material. It becomes a method, a way of thinking, a way of acknowledging by exploring reality and inviting the reader to take part in a dialogue. However, one condition is that the essay is something more than just a well-told story and some entertaining anecdotes. The quality of the essay is realised when the writer can discover the large in the little and the generic within the special case. The well written scientific essay makes the reader come to realize that the unique experience and the particular interpretations described by the writer have qualities that are genuinely significant for our common effort in comprehending reality.

Far from just being a textual category, a genre represents the very social-semiotic framework for communication, making the realm in which linguistic resources are intertwined with and work together with social conventions and certain ways of acknowledging. The informal essay offers interesting possibilities to enhance the reportoire of scientific relevant and valuable impressions and expressions, both for researchers and students. 


\section{References:}

Berge, Kjell Lars (2003). "The scientific text genres as social actions: text theoretical reflections on the relations between context and text in scientific writing". In: K. Fløttum \& F. Rastier (eds.) Academic discourse - multidisciplinary approaches. Oslo: Novus. 141-157

Dunn, R., Griggs, S.A., Olson, J., Gorman, B. \& Beasley, M. (1995). A meta analytic validation of the Dunn and Dunn Learning Style model. Journal of Educational Research. 88(6), p. 353 - 364.

Dunn, Rita and Kenneth J (1972). Practical approaches to individualizing instruction: contracts and other effective teaching strategies. West Nyack, N.Y. : Parker

Halliday, M. A. K. (1985). An introduction to functional grammar. London : Arnold

Linell, Per (1995). Approaching dialogue : talk and interaction in dialogical perspectives. Linköping : Linköpings universitet

Wells, G. (1999). Dialogic inquiry: Towards a sociocultural practice and theory of education. New York: Cambridge University Press. 